\title{
A Computational Method for $n$-Dimensional Laplace Transforms Involved with Fourier Cosine Transform
}

\author{
Jafar Saberi-Nadjafi ${ }^{1,2}$ \\ ${ }^{1}$ Department of Applied Mathematics, School of Mathematical Sciences, Ferdowsi University of Mashhad, Mashhad, Iran \\ ${ }^{2}$ The Center of Excellence on Modeling and Control Systems (CEMCE), Ferdowsi University of Mashhad, Mashhad, Iran \\ Correspondence should be addressed to Jafar Saberi-Nadjafi; najafil41@gmail.com
}

Received 24 May 2013; Accepted 9 July 2013

Academic Editors: Y.-D. Kwon and G. Mishuris

Copyright (C) 2013 Jafar Saberi-Nadjafi. This is an open access article distributed under the Creative Commons Attribution License, which permits unrestricted use, distribution, and reproduction in any medium, provided the original work is properly cited.

In 2007, the author published some results on $n$-dimensional Laplace transform involved with the Fourier sine transform. In this paper, we propose some new result in $n$-dimensional Laplace transforms involved with Fourier cosine transform; these results provide few algorithms for evaluating some $n$-dimensional Laplace transform pairs. In addition, some examples are also presented, which explain the useful applications of the obtained results. Therefore, one can produce some two- and three- as well as $n$ dimensional Laplace transforms pairs.

\section{Introduction and Preliminaries}

Before a lunching into the main part of the paper, we define some notations and terminologies which will remain standard. The classification $n$-dimensional Laplace transform under consideration for a function $f(\bar{t})$ is a function $F(\bar{s})$ through the relation

$$
\begin{aligned}
F(\bar{s}) & =\mathscr{L}\{f(\bar{t}) ; \bar{s}\} \\
& =\int_{0}^{\infty} \int_{0}^{\infty} \cdots \int_{0}^{\infty} \exp (-\bar{s} \cdot \bar{t}) f(\bar{t}) p_{n}(d \bar{t}),
\end{aligned}
$$

where $\bar{t}=\left(t_{1}, t_{2}, \ldots, t_{n}\right), \bar{s}=\left(s_{1}, s_{2}, \ldots, s_{n}\right), \bar{s} \cdot \bar{t}=\sum_{i=1}^{n} s_{i} t_{i}$, and $p_{n}(d \bar{t})=\prod_{k=1}^{n} d t_{k}$. The domain of definition of $F$ is the set of all points $\bar{s} \in \mathbb{C}^{n}$ such that the integral in (1) is convergent. Instead of the $n$-dimensional Laplace transform (1), sometimes we calculate the so-called $n$-dimensional Carson-Laplace transform:

$$
F(\bar{s})=p_{n}(\bar{s}) \int_{0}^{\infty} \int_{0}^{\infty} \cdots \int_{0}^{\infty} \exp (-\bar{s} \cdot \bar{t}) f(\bar{t}) p_{n}(d \bar{t}) .
$$

Symbolically, we denote the pairs $F(\bar{s})$ and $f(\bar{x})$ by the following operational relation:

$$
F(\bar{s}) \frac{n}{n} f(\bar{t}) \quad \text { or } \quad f(\bar{t}) \frac{n}{n} F(\bar{s}) .
$$

In this notation, some of the formulas become more simple.

We denote (3) in one-dimensional case by the following:

$$
F(s) \doteqdot f(x) .
$$

Now, if the $n$-dimensional Laplace transform is known, its inverse is given by the following:

$$
f(\bar{t})=\frac{1}{(2 \pi i)^{n}} \int_{\mathrm{Br}} \int_{\mathrm{Br}} \cdots \int_{\mathrm{Br}} \exp (\bar{s} \cdot \bar{t}) F(\bar{s}) p_{n}(d \bar{s}) .
$$

Herein, $\mathrm{Br}$ designates the appropriate Bromwich contour integral in the plane of integration.

For brevity, we will also use the following notation throughout this paper.

Let $\overline{t^{\nu}}=\left(t_{1}^{\nu}, t_{2}^{\nu}, \ldots, t_{n}^{\nu}\right)$ for any real exponent $v$, and let $p_{k}(\bar{t})$ be the $k$ th symmetric polynomial in the component $t_{k}$ of $\bar{t}$. Then we denote 
(i) $p_{1}\left(\overline{t^{\nu}}\right)=\sum_{i=1}^{n} t_{i}^{\nu}$,

(ii) $p_{n}\left(\overline{t^{\nu}}\right)=\prod_{i=1}^{n} t_{i}^{\nu}$.

The difficulties in obtaining multiple direct or inversion Laplace transforms (1) or (5) that appear in problems of physics and engineering lead to continuous efforts in expanding the transform tables for directs and designing algorithms generating new inverses transforms from known ones. While such tables are available, the actual evaluation of the direct and inversion integral is obviated and the solution of boundary value problems in several variables and some partial differential equations is reduced to a relatively routine procedure. For more details on this subject see [1-16].

\section{The Main Results}

In this section we state and give proof for our main theorems, which give some new $n$-dimensional Laplace transforms pairs for arbitrary nonnegative integer $n \geq 2$.

Theorem 1. Suppose that

(i) $\mathscr{L}\{g(x) ; s\}=F(s)$

(ii) $\mathscr{L}\{F(x) ; s\}=f(s)$

(iii) $\mathscr{L}\left\{x^{(n-1) / 2} g(x) ; s\right\}=\phi_{(n-1) / 2}(s), n=2,3, \ldots, N$.

Also, let $F_{c}(s)$ be the Fourier cosine transform of $f\left(x^{2}\right)$, and let $\exp (-s x-x t) g(x)$ belong to $L_{1}[(0, \infty) \times(0, \infty)]$. Then

$$
\begin{gathered}
\mathscr{L}_{n}\left\{p_{n}\left(\overline{x^{-2 / 3}}\right) \phi_{(n-1) / 2}\left(\frac{1}{4} p_{1}\left(\overline{x^{-1}}\right)\right) ; \bar{s}\right\} \\
=2^{n+1} \pi^{(n-2) / 2} F_{c}\left[p_{1}\left(\overline{s^{1 / 2}}\right)\right],
\end{gathered}
$$

provided the Laplace transform of functions $g(x), F(x)$ and $x^{(n-2) / 2} g(x), n \geq 2$, exist and the integrals in the left side of (6) also exist in every variable.

Proof. By using the assumptions (i) and (ii) together, we get

$$
f(s)=\int_{0}^{\infty} \exp (-s x)\left[\int_{0}^{\infty} \exp (-x t) g(t) d t\right] d x .
$$

Now, interchanging the order of the integrals on the right side of (7) due to the Fubini's theorem [17] evaluating the inner integral and next by replacing $s$ by $v^{2}$ in the resulting equation, we have

$$
f\left(v^{2}\right)=\int_{0}^{\infty} g(t) \frac{1}{v^{2}+t} d t
$$

From (8) we can easily obtain

$$
\int_{0}^{\infty} f\left(\nu^{2}\right) \cos (s v) d v=\int_{0}^{\infty} g(t)\left[\int_{0}^{\infty} \frac{\cos (s v)}{v^{2}+t} d \nu\right] d t
$$

Evaluating the inner integral in the rightside of (9), we get

$$
\int_{0}^{\infty} f\left(v^{2}\right) \cos (s v) d v=\frac{\pi}{2} \int_{0}^{\infty} t^{-1 / 2} g(t) \exp \left(-t^{1 / 2} s\right) d t
$$

By the assumption, (10) can be rewritten as

$$
F_{c}(s)=\frac{\pi}{2} \int_{0}^{\infty} t^{-1 / 2} g(t) \exp \left(-t^{1 / 2} s\right) d t
$$

Next, we replace $s$ by $p_{1}\left(\overline{s^{1 / 2}}\right)$ in (11) and multiply both sides of the resulting relation by $p_{n}(\bar{s})$, in order to obtain

$$
\begin{aligned}
p_{n} & (\bar{s}) F_{c}\left[p_{1}\left(\overline{s^{1 / 2}}\right)\right] \\
& =\frac{\pi}{2} p_{n}(\bar{s}) \int_{0}^{\infty} t^{-1 / 2} g(t) \exp \left[-t^{1 / 2} p_{1}\left(\overline{s^{1 / 2}}\right)\right] d t .
\end{aligned}
$$

Now, we use the following operational relation which is given in $[18]$, in (12)

$$
s_{i} \exp \left[-a s_{i}^{1 / 2}\right] \doteqdot \frac{a x_{1}^{-3 / 2}}{\pi^{1 / 2}} \exp \left[-\frac{a^{2}}{4 x_{1}}\right]
$$

for $i=1,2, \ldots, n,(12)$ reads

$$
p_{n}(\bar{s}) F_{c}\left[p_{1}\left(\overline{s^{1 / 2}}\right)\right] \underset{n}{\frac{n}{n}} \frac{p_{n}\left(\overline{x^{-3 / 2}}\right)}{2^{n+1} \pi^{(n-2) / 2}} \phi_{(n-1) / 2}\left(\frac{p_{1}\left(\overline{x^{-1}}\right)}{4}\right) .
$$

Therefore,

$$
\begin{gathered}
\mathscr{L}_{n}\left\{p_{n}\left(\overline{x^{-3 / 2}}\right) \phi_{(n-1) / 2}\left(\frac{1}{4} p_{1}\left(\overline{x^{-1}}\right)\right) ; \bar{s}\right\} \\
=2^{n+1} \pi^{(n-2) / 2} F_{c}\left[p_{1}\left(\overline{s^{1 / 2}}\right)\right] .
\end{gathered}
$$

This completes the proof.

Theorem 2. Suppose all conditions given in Theorem 1 hold true but replace the condition (iii) by the following:

$$
\left(\text { iii) }^{\prime} \mathscr{L}\left\{x^{-1 / 2} g(x) ; s\right\}=\phi_{-1 / 2}(s) .\right.
$$

Then

$$
\begin{aligned}
\mathscr{L}_{n}\left\{p_{n}\left(\overline{x^{-1 / 2}}\right) \phi_{-1 / 2}\left(\frac{1}{4} p_{1}\left(\overline{x^{-1}}\right)\right) ; \bar{s}\right\} \\
=\frac{\pi^{(n-2) / 2}}{2^{1 / 2}} p_{n}\left(\overline{s^{-1 / 2}}\right) F_{c}\left[p_{1}\left(\overline{s^{1 / 2}}\right)\right] .
\end{aligned}
$$

Proof. The proof of Theorem 2 is similar to that of Theorem 1 , and we therefore omit it.

The following examples will illustrate the applications of Theorems 1 and 2. We will consider the function $g$ to be an elementary or some special function to construct certain functions with $n$ variables, and we calculate their Laplace transforms, using Theorems 1 and 2. The first two examples are related to Theorem 1, and Examples 3 and 4 illustrate the application of Theorem 2 . 


\section{3. $n$-Dimensional Examples}

Example 1. Suppose $g(x)=x^{-v / 2}$ for $-1<\operatorname{Re} v<0$, so that using [18] and assumptions (i)-(iii) in Theorem 1, we get the following:

$$
\begin{gathered}
F(s)=\frac{\Gamma(1-(\nu / 2))}{s^{1-(v / 2)}}, \quad \operatorname{Re} s>0, \\
f(s)=\frac{\Gamma(1-(\nu / 2)) \Gamma(\nu / 2)}{s^{\nu / 2}}, \quad \operatorname{Re} s>0, \\
\phi_{(n-1) / 2}(s)=\frac{\Gamma((n-\nu+1) / 2)}{s^{(n-v+1) / 2}}, \quad \operatorname{Re} s>0 .
\end{gathered}
$$

Next, using a formula given in [19], we obtain

$$
F_{c}(s)=\frac{\pi^{2}}{\Gamma(\nu) \sin \pi \nu} s^{\nu-1}
$$

Hence from Theorem 1, we have

$$
\begin{gathered}
\mathscr{L}_{n}\left\{\frac{p_{n}\left(\overline{x^{-3 / 2}}\right)}{\left[p_{1}\left(\overline{x^{-1}}\right)\right]^{(n-\nu+1) / 2}} ; \bar{s}\right\} \\
=\frac{2^{n-1} \pi^{(n+2) / 2}}{\Gamma(\nu) \sin \pi \nu}\left[p_{1}\left(\overline{s^{1 / 2}}\right)\right]^{\nu-1}, \\
a>0, \quad \operatorname{Re}\left[p_{1}\left(\overline{s^{1 / 2}}\right)\right]>0 .
\end{gathered}
$$

Example 2. Let us assume $g(x)=x^{1 / 4} J_{1 / 2}\left(2 a^{1 / 2} x^{1 / 2}\right), a>0$. Using formulas given in [18], we obtain

$$
\begin{gathered}
F(s)=a^{1 / 4} s^{-3 / 2} e^{-a / s}, \quad \operatorname{Re} s>0, \\
f(s)=\frac{\pi^{1 / 2}}{a^{1 / 4}} e^{-2 a^{1 / 2} s^{1 / 2}}, \quad \operatorname{Re} s>0, \\
\phi_{(n-1) / 2}(s)=\frac{n \Gamma(n) \pi^{-1 / 2} a^{1 / 4}}{s^{(n / 2)+1}}{ }_{1} F_{1}\left[\frac{n}{2}+1 ; \frac{3}{2} ;-\frac{a}{s^{1 / 2}}\right], \\
\operatorname{Re} s>0,
\end{gathered}
$$

where by ${ }_{1} F_{1}[\cdot ; \cdot ; \cdot]$ we mean generalized hypergeometric function.

Next, the formula given in [19] yields

$$
F_{c}(s)=\frac{2 \pi^{1 / 2} a^{1 / 4}}{4 a+s^{2}}
$$

Using Theorem 1, we get

$$
\begin{aligned}
& \mathscr{L}_{n}\left\{\frac{p_{n}\left(\overline{x^{-3 / 2}}\right)}{\left(p_{1}\left(\overline{x^{-1}}\right)\right)^{(n / 2)+1}}\right. \\
& \left.\quad \times{ }_{1} F_{1}\left[\frac{n}{2}+1 ; \frac{3}{2} ;-\frac{2 a}{\left(p_{1}\left(\overline{x^{-1}}\right)\right)^{1 / 2}}\right] ; \bar{s}\right\} \\
& =\frac{\pi^{n / 2}}{n \Gamma(n)} \cdot \frac{1}{4 a+\left[p_{1}\left(\overline{s^{1 / 2}}\right)\right]^{2}}, \\
& a>0, \operatorname{Re}\left[p_{1}\left(\overline{s^{1 / 2}}\right)\right]>0 .
\end{aligned}
$$

Example 3. Assuming $g(x)=\sin a x^{1 / 2}, a>0$, and using formulas given in [18], we get the following:

$$
\begin{gathered}
F(s)=\frac{a \pi^{1 / 2}}{2} s^{-3 / 2} e^{-a^{2} / 4 s}, \quad \operatorname{Re} s>0, \\
f(s)=\frac{4}{a^{2}} e^{-a s^{1 / 2}}, \quad \operatorname{Re} s>0, \\
\phi_{-1 / 2}(s)=\frac{\pi^{1 / 2}}{s^{1 / 2}} e^{-a^{2} / 4 s} \operatorname{erfi}\left(\frac{a}{2 s^{1 / 2}}\right), \quad \operatorname{Re} s>0,
\end{gathered}
$$

where

$$
\operatorname{erfi}(x)=-i \operatorname{erfi}(i x)=\frac{2}{\pi^{1 / 2}} \int_{0}^{x} e^{t^{2}} d t
$$

Afterward, with a formula given in [19], we obtain

$$
F_{c}=\frac{a^{3}}{4\left(a^{2}+s^{2}\right)}
$$

Therefore, from Theorem 2, we have

$$
\begin{gathered}
\mathscr{L}_{n}\left\{\frac{p_{n}\left(\overline{x^{-1 / 2}}\right)}{\left.\left(p_{1}\left(\overline{x^{-1}}\right)\right)^{1 / 2} e^{\left(-a^{2} / p_{1}\left(\overline{x^{-1}}\right)\right.}\right) \text { erfi }}\right. \\
\left.\times\left[\frac{a}{\left(p_{1}\left(\overline{x^{-1}}\right)\right)^{1 / 2}}\right] ; \bar{s}\right\} \\
=\frac{a^{3} \pi^{(n-2) / 2}}{2^{5 / 2}} \cdot \frac{p_{n}\left(\overline{s^{1 / 2}}\right)}{a^{2}+p_{1}^{2}\left(\overline{s^{1 / 2}}\right)} \\
a>0, \operatorname{Re}\left[p_{1}\left(\overline{s^{1 / 2}}\right)\right]>0 .
\end{gathered}
$$


Example 4. Suppose that $g(x)=x^{-1 / 4} \exp (a x)$. Then with the aid of formulas given in $[18,20]$, we obtain

$$
\begin{gathered}
F(s)=\frac{\Gamma(3 / 4)}{(s-a)^{3 / 4}}=\frac{\pi \sqrt{2}}{\Gamma(1 / 4)(s-a)^{3 / 4}}, \quad \text { Re } s>a, \\
f(s)=\frac{1}{\sqrt{2} \pi s^{1 / 4}} \exp (-a s), \quad \text { Re } s>0, \\
\phi_{-1 / 2}(s)=\frac{\Gamma(1 / 4)}{(s-a)^{1 / 4}}, \quad \text { Re } s>-a .
\end{gathered}
$$

Now, by using a formula given in [19], we get

$$
F_{c}(s)=\frac{\sqrt{s}}{4 \sqrt{a}} \exp \left(-\frac{s^{2}}{8 a}\right) I_{-1 / 4}\left(\frac{s^{2}}{8 a}\right),
$$

where by $I_{-1 / 4}(\cdot)$ we mean the modified Bessel function of the first kind.

Putting the above relations into (16), we arrive at the following:

$$
\begin{aligned}
\mathscr{L}_{n}\left\{\frac{p_{n}\left(\overline{x^{-1 / 2}}\right)}{\left[(1 / 4) p_{1}\left(\overline{x^{-1}}\right)-a\right]^{1 / 4}} ; \bar{s}\right\} \\
=\frac{\pi^{(n-1) / 2}}{4 \Gamma(1 / 4) \sqrt{2} a} p_{n}\left(\overline{s^{-1 / 2}}\right) \sqrt{p_{1}\left(\overline{s^{1 / 2}}\right)} \\
\quad \times \exp \left[-\frac{p_{1}^{2}\left(\overline{s^{1 / 2}}\right)}{8 a}\right] I_{-1 / 4}\left[\frac{p_{1}^{2}\left(\overline{s^{1 / 2}}\right)}{8 a}\right], \\
a>0 .
\end{aligned}
$$

\section{Conclusion}

In this paper, we presented and proved two main theorems concerned with $n$-dimensional Laplace transform involved with the Fourier cosine transform. These theorems provide few algorithms for evaluating some $n$-dimensional Laplace transform pairs. The formulas are obtained in Examples 14 all of which are new results both in two-dimensional and in the corresponding results in $n$-dimensional Laplace transform pairs. Several other new results can be obtained using these algorithms.

\section{Acknowledgment}

This research was supported by a Grant from Ferdowsi University of Mashhad (no. MA88062SAB).

\section{References}

[1] A. Aghili and B. Salkhorde-Moghadam, "Laplace transform pairs of $n$-dimensions and a wave equation," International Mathematical Journal, vol. 5, no. 4, pp. 377-382, 2004.

[2] A. Aghili and B. Salkhordeh Moghaddam, "Laplace transform pairs of $n$-dimensions and second order linear partial differential equations with constant coefficients," Annales Mathematicae et Informaticae, vol. 35, pp. 3-10, 2008.
[3] A. Aghili and B. Salkhordeh Moghaddam, "Laplace transform pairs of $n$-dimensions and a wave equation," International Journal of Mathematics, vol. 5, no. 4, pp. 377-382, 2004.

[4] Y. A. Brychkov, H. J. Glaeske, A. P. Prudnikov, and V. K. Tuan, Multidimensional Integral Transformations, Gordon and Breach Science, Philadelphia, Pa, USA, 1992.

[5] R. S. Dahiya, "Computation of $n$-dimensional Laplace transforms," Rendicontidi Mathematica, vol. 8, no. 3, pp. 805-813, 1975.

[6] R. S. Dahiya, "Computation of $n$-dimensional Laplace transforms," Journal of Computational and Applied Mathematics, vol. 3, no. 3, pp. 185-188, 1977.

[7] R. S. Dahiya, "Laplace transform pairs of $n$-dimensions," International Journal of Mathematics and Mathematical Sciences, vol. 8, no. 3, pp. 449-454, 1985.

[8] R. S. Dahiya and M. Vinayagamoorthy, "Laplace transform pairs of $n$-dimensions and heat conduction problem," Mathematical and Computer Modelling, vol. 13, no. 10, pp. 35-50, 1990.

[9] R. S. Dahiya and J. Saberi-Nadjafi, "Theorems on $n$-dimensional Laplace transforms and their applications," in Proceedings of the 15th Annual Conference of Applied Mathematics, University of Central Oklahoma, Electronice Journal of Differential Equations, vol. 2, pp. 61-74, 1999.

[10] J. Debnath and R. S. Dahiya, "Theorems on multidimensional Laplace transform for solution of boundary value problems," Computers \& Mathematics with Applications, vol. 18, no. 12, pp. 1033-1056, 1989.

[11] T. A. Estrin and T. J. Higgins, "The solution of boundary value problems by multiple Laplace transformations," Journal of the Franklin Institute, vol. 252, no. 2, pp. 153-167, 1951.

[12] J. Saberi-Nadjafi, "Theorems on $n$-dimensional inverse Laplace transformations," in Proceedings of the 8th Annual Conference on Applied Mathematics, pp. 317-330, Edmond, Okla, USA, 1992.

[13] J. Saberi-Nadjafi and R. S. Dahiya, "Certain theorems on $n$ dimensional Lplace transformations and their applications," in Proceedings of the 8th Annual Conference on Applied Mathematics, pp. 245-285, Edmond, Okla, USA, 1992.

[14] J. Saberi-Nadjafi, $n$-dimensional Laplace transformaton and their applications in partial differential equations [Ph.D. Dissertation], Iowa State University, Ames, Iowa, USA, 1993.

[15] J. Saberi-Nadjafi and R. S. Dahiya, “Theorems on $n$-dimensional Laplace transformations involving the solution of wave equations," Integral Transforms and Special Functions, vol. 15, no. 4, pp. 337-358, 2004.

[16] J. Saberi-Nadjafi, "Some remarks on $n$-dimensional Laplace transforms," Italian Journal of Pure and Applied Mathematics, no. 21, pp. 163-168, 2007.

[17] C. Chapman Pugh, Real Mathematical Analysis, Springer, New York, NY, USA, 2002.

[18] A. P. Prudnikov, Y. A. Brychkov, and O. I. Marichev, Integrals and Series, vol. 4 of Direct Laplace transforms, Gordon and Breach Science, New York, NY, USA, 1992.

[19] H. Bateman, Tables of Integral Transformations, vol. 1, McGrawHill Book, 1954.

[20] A. P. Prudnikov, Y. A. Brychkov, and O. I. Marichev, Integralsand Series, vol. 5 of Inverse Laplace transforms, Gordon and Breach Science, New York, NY, USA, 1992. 


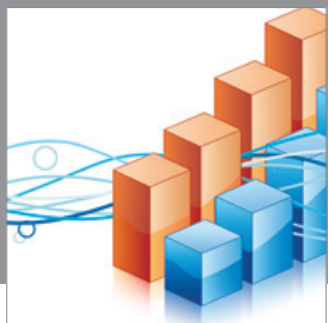

Advances in

Operations Research

mansans

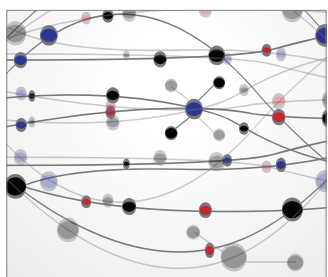

The Scientific World Journal
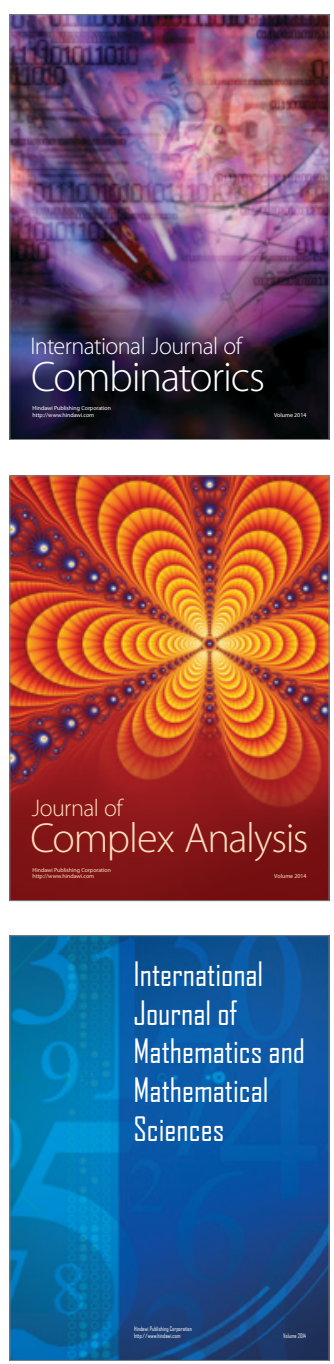
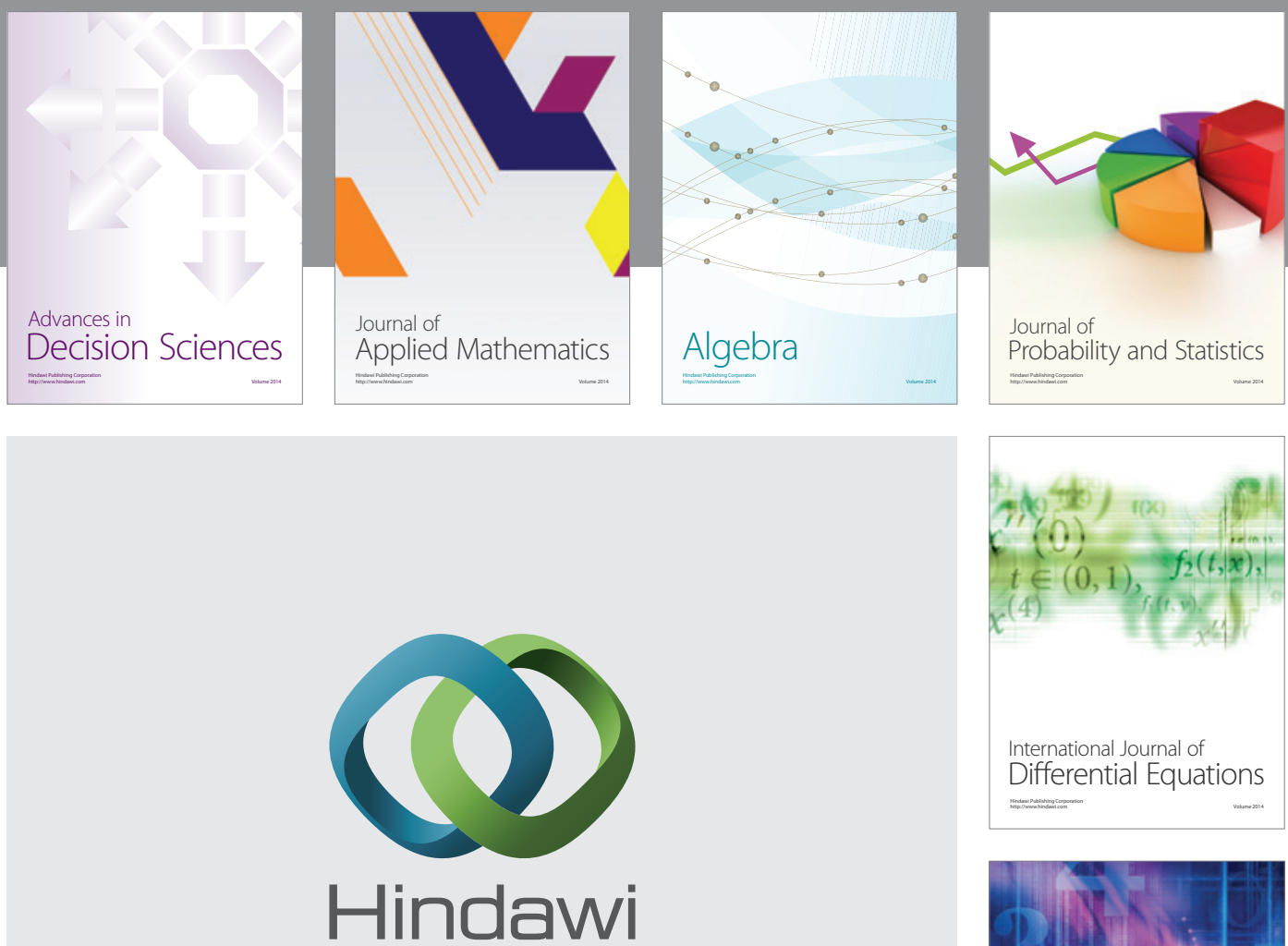

Submit your manuscripts at http://www.hindawi.com
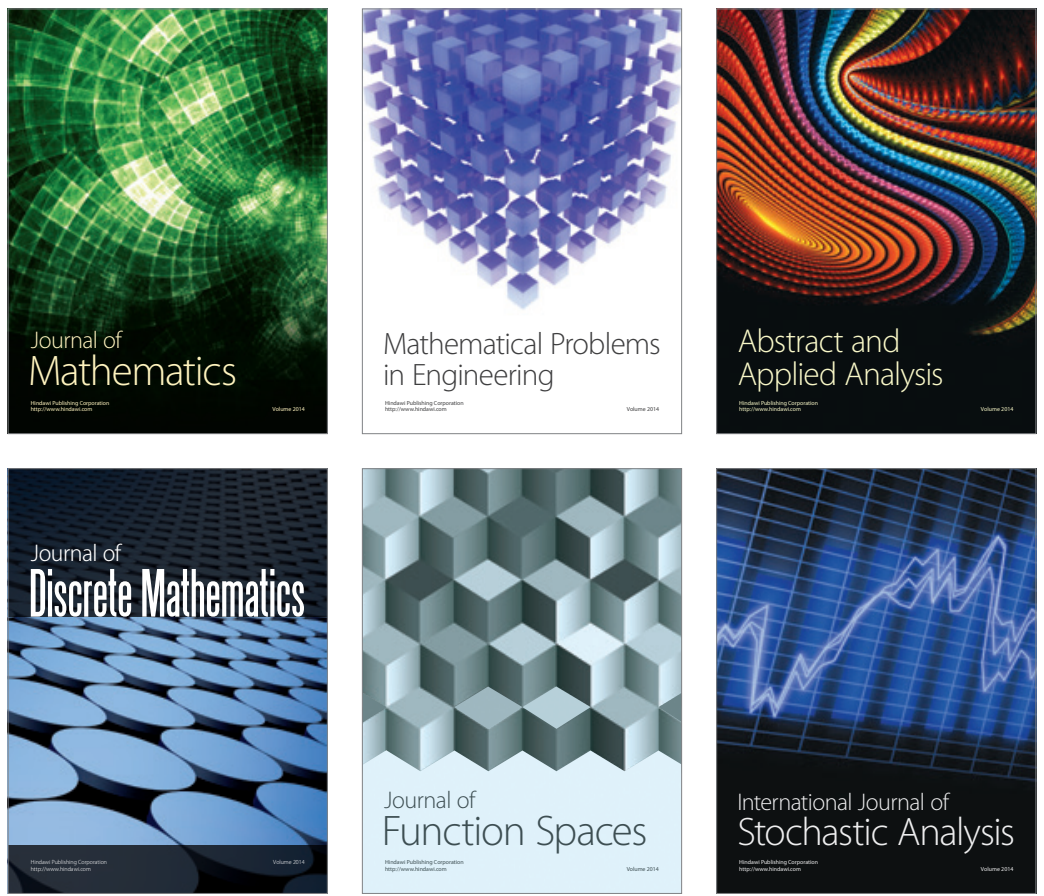

Journal of

Function Spaces

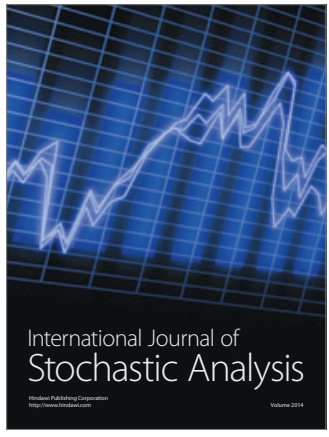

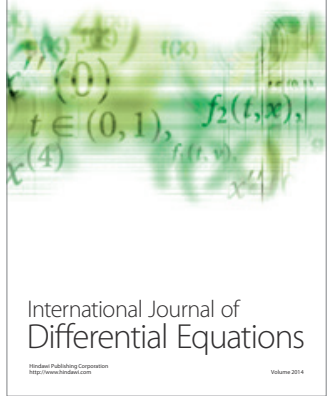
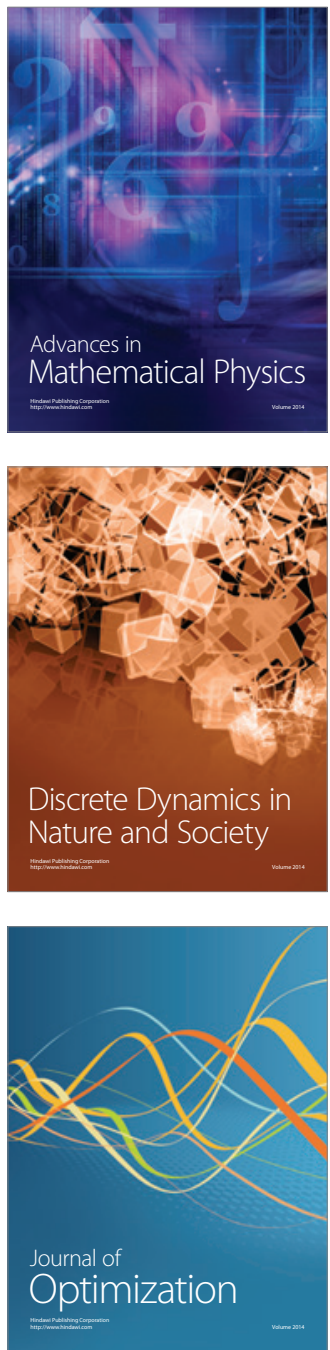